\title{
IA-1 APPLICATIONS AND CHALLENGES FOR HIGH RESOLUTION AIRBORNE SURVEYS IN POPULATED AREAS
}

M.K. LEE ${ }^{1}$, R.J. PEART ${ }^{1}$, R.J. CUSS $^{1}$, D.G. JONES ${ }^{1}$, D. BEAMISH ${ }^{1}$ and J. VIRONMAKI ${ }^{2}$

${ }^{1}$ British Geological Survey, Keyworth, Nottingham, NG12 5GG, UK

${ }^{2}$ Geological Survey of Finland (GTK)

\section{Introduction}

The role of high resolution airborne surveys is extending well beyond the traditional areas of mineral and hydrocarbons exploration and regional mapping, which were often focused on the relatively unpopulated and remote regions of the world. As an increasing proportion of the Earth's surface is affected by human activity, airborne survey techniques are being adapted and applied to development-related issues in more populated areas. The value of radiometric and electromagnetic data (in particular) has been recognised for addressing a range of environmental issues and, more generally, airborne surveys are seen as an effective, non-invasive means of providing multiple development-related geoscience data sets in a single operation. These new applications present many operational, processing and interpretation challenges. This paper provides an overview of some of the issues addressed in this Special Session on 'The role of high resolution airborne surveys in environmental monitoring and sustainable development of resources'.

\section{Applications}

In the context of this discussion, 'populated areas' range from urban and industrial centres to open farm land and semi-wild landscapes with scattered populations. The common factor is that the human population is having an increasingly significant impact on the land by using it for industrial and residential development, intensive agriculture, recreation and extraction of natural resources. There is, therefore, a growing need to identify natural and man-made hazards and to provide an environmental baseline for land-use planning. Furthermore, the exploitation of natural resources is increasingly only allowed within the framework of sustainable development principles. This entails planning the full life-cycle of resource exploitation (exploration, preextraction baseline observations, extraction impact monitoring, closure and post-closure monitoring) in order to minimise environmental and human impacts. Such 'holistic' planning, in turn, requires a more detailed definition of surface and subsurface properties and a greater understanding of the interaction between human activity and the geosphere.

High resolution airborne surveys are usually carried out along closely-spaced (50-400m) flight lines at a terrain clearance of $<100 \mathrm{~m}$, acquiring various combinations of magnetic, radiometric (spectral gamma) and electro-magnetic (EM) data. Together, these represent an extremely powerful and integrated series of data sets for addressing a range of environmental and development-related issues and characterising the key properties of the surface and subsurface geology. These include: 
- defining the distribution of natural and anthropogenic radionuclides (including radioactive industrial waste)

- defining baseline levels of radioactivity against which any future radioactive contamination can be assessed

- identifying regions prone to high radon levels

- identifying pollution of land and groundwater from industrial and waste sites

- defining the character of surface and shallow subsurface geology to support decision making on a wide spectrum of land use planning, including waste disposal, resource exploitation and geo-hazard assessment

- providing new data to support sustainable development of coal, gas, oil, metalliferous minerals and construction minerals throughout all stages of the resource life cycle (exploration, extraction and closure).

- contributing to a better understanding of the surface and concealed geology

In 1998 the British Geological Survey (BGS) carried out the UK's first regional high-resolution survey (HiRES-1) over part of the English Midlands in order to demonstrate the value of airborne surveys and build a case for a long-term programme. Magnetic, radiometric and VLFEM data were collected over a $200 \times 70 \mathrm{~km}$ area by World Geoscience (UK) Ltd (now part of Fugro Airborne Surveys Ltd) at a line spacing of $400 \mathrm{~m}$ (200 m in selected areas) and a flying height of $90 \mathrm{~m}$ (Figure 1). In 1999 a smaller trial survey was carried out in collaboration with the Geological Survey of Finland (GTK). The objective in this case was to test the effectiveness of GTK's airborne system (comprising magnetic, radiometric and dual-frequency EM capability) over selected environmental targets in the East Midlands. The results from these surveys are discussed in other papers in this session, but it is worth noting in particular the effectiveness of high resolution radiometric data for characterising important variations in radioactivity (Figure 2 ) and the effectiveness of the EM technique for investigating potential pollution from mine waste and land-fill sites (Figure 3).

\section{Technical and operational challenges}

The recent surveys carried out in the UK highlighted a number of difficulties and challenges related to acquiring, processing and interpreting high resolution airborne data in 'populated' areas.

\section{The flying environment and air safety}

Safety must be the paramount concern for any survey. Countries with a relatively high population density present a particularly challenging set of flying condition for high resolution survey operators. The skies are often relatively crowded and the survey has to compete with a range of commercial, recreational and military uses. In addition, weather and flying restrictions may vary from area to area on a daily basis, forcing the operator to collect data in a less systematic way than would be the case in remote areas.

Possibly the most challenging aspect, however, is the need to observe rigorous regulations on flying height whilst maximising anomaly resolution. In the UK, the Civil Aviation Authority usually permit flying at $90 \mathrm{~m}$ terrain clearance in rural areas (occasionally lower in some 
circumstances), but in urban areas clearance must be greater that $245 \mathrm{~m}$. In most areas of the UK, therefore, the pilot will need to vary the terrain clearance along each line and keep a close watch for potential hazards (other air traffic, electricity power lines, etc).

In practice, flying heights for the HiRES- 1 survey varied between around 90-370m and humidity varied between $35-93 \%$. The resulting data required particularly careful processing to correct for these variations. Radiometric and EM data are very sensitive to changes in terrain clearance while adjacent flight lines may have been acquired in different weather conditions (saturated ground will reduce the gamma count). Nevertheless, when these factors are recognised and taken into account, consistent and effective data can be achieved.

\section{Cultural noise}

Cultural noise from buildings and the transport infrastructure interferes with, and masks, both natural and environmental signals. The processing of magnetic data represents a particularly demanding challenge, especially if the terrain clearance varies along each line as indicated above. Digital filtering of magnetic data can remove a proportion of the isolated cultural anomalies but its effectiveness is severely limited because (i) closely-spaced cultural anomalies overlap and interfere with each other, and (ii) if the terrain clearance varies along each line there is significant spectral overlap between anomalies due to man-made and shallow natural sources. BGS has found that the only really effective method of removing cultural noise is to identify the spatial correlation of anomalies and features on the ground (from maps, aerial photographs or the in-flight video recordings) within a GIS environment, and then to edit-out the cultural anomalies as necessary (see Figure 4).

\section{Separating natural and environmental sources}

There is a complex relationship between natural, man-made and environmentally-significant anomalies, and the ability to detect an anomaly from an artificial source will vary according to the host geology. For example, a closed and forgotten land-fill site may contain significant quantities of iron that gives rise to a magnetic anomaly very similar in character (at first sight) to that from shallow magnetic rocks. Ground conductivity varies considerably between geological formations; a highly conductive zone around a land-fill site within a resistive host rock might indicate a genuine pollution plume, whereas the same value around a site in a more conductive

host rock might be insignificant. Particular care is needed in analysing radiometric data; $\mathrm{U}$ or Th counts may be 'high' in geological terms but still well below the levels that might pose a significant risk. The challenge in all of these cases is to develop and apply the appropriate analysis and interpretation techniques tuned specifically to addressing development-related problems.

\section{Policy and funding challenges}

The fundamental case for acquiring high resolution airborne data over the more populated parts of the world (e.g. most of Europe) is to provide important new layers of information to underpin decision making on land-use planning, the detection and mitigation of natural and man-made geo-hazards and the sustainable development of natural resources.

Airborne surveys are not cheap (say \$5-50 per km depending on the platform, survey configuration and area) and it is often difficult to justify the cost of systematic coverage over entire countries on the basis of a single application (e.g. resources exploration). However, when 
the value of the data across all sectors is taken together, the case becomes compelling and cost effective. The challenge for the geoscience community is, therefore, to demonstrate the vital contribution that the data can make to a wide range of environmental, planning and resource sectors, with the aim of securing the necessary funding for wide-spread coverage. Arguably, the absence of high resolution airborne data now represents one of the most serious gaps in the environmental and geoscience knowledge base in many parts of the world.

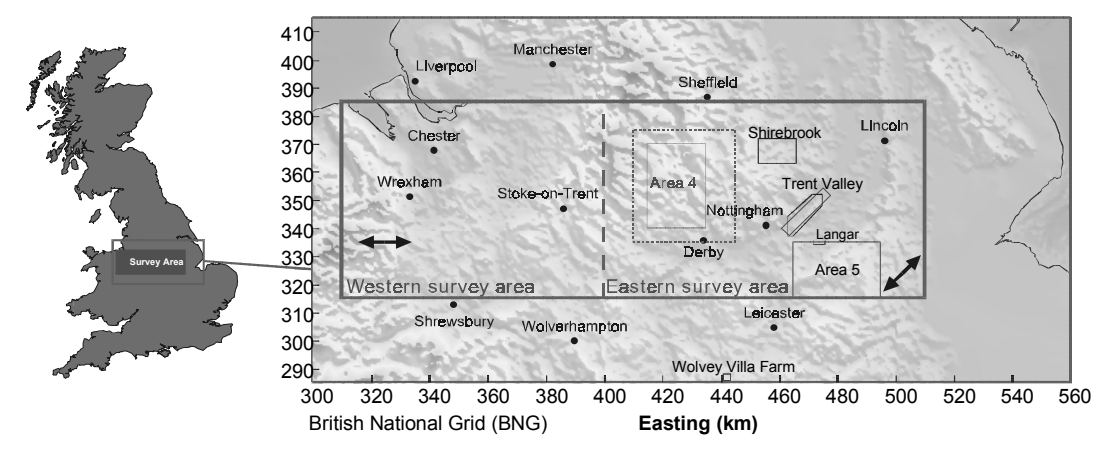

Figure 1. Location of recent UK high resolution surveys (HiRES-1 and GTK trial)
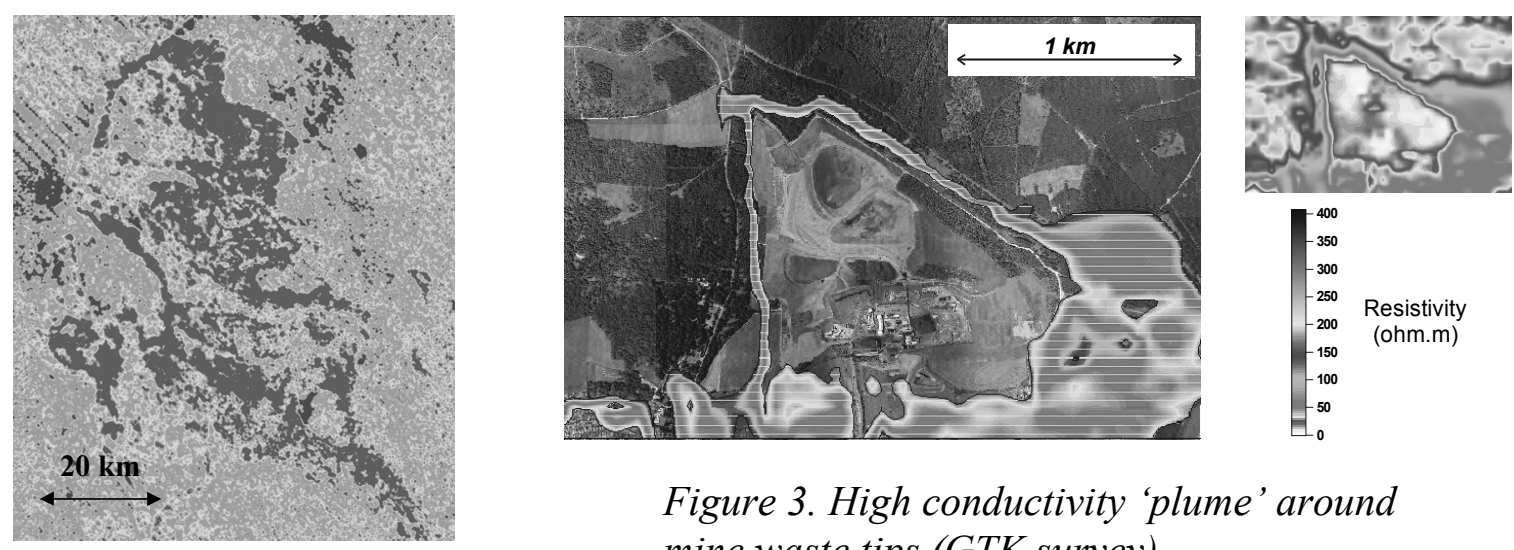

Figure 3. High conductivity 'plume' around mine waste tips (GTK survey)

Figure 2. Relatively high U values (dark tones) in the English Peak District indicating potential radon risk (HiRES-1)

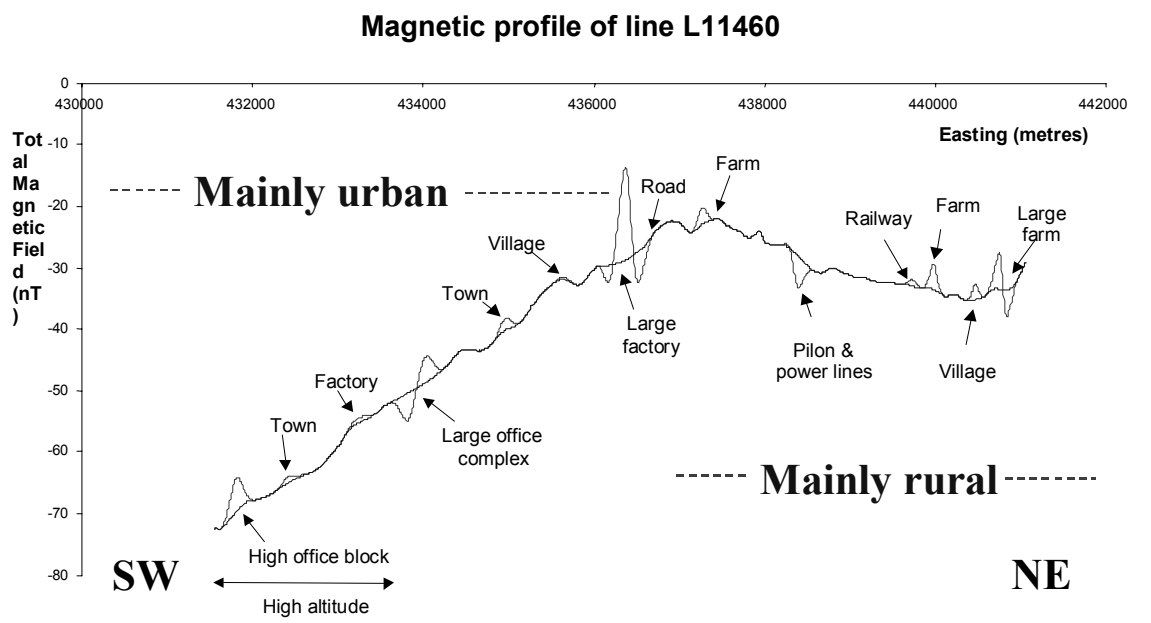

Figure 4. Overlap between natural and artificial magnetic anomalies (HiRES-1) 